\title{
Efficacy and tolerability of propiverine hydrochloride extended-release compared with immediate-release in patients with neurogenic detrusor overactivity
}

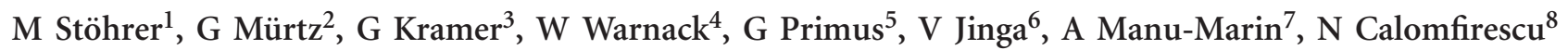 \\ and G Strugala ${ }^{2}$
}

Study design: Double-blind, randomised, multicentre study.

Objectives: Efficacy and tolerability of propiverine extended-release (ER) compared with immediate-release (IR) were evaluated in patients with proven neurogenic detrusor overactivity (NDO).

Setting: Six Spinal Cord Injury Units located in Austria, Germany and Romania.

Methods: Propiverine ER $45 \mathrm{mg}$ s.i.d. or IR $15 \mathrm{mg}$ t.i.d. were administered in patients with proven NDO. Outcomes were assessed at baseline (V1), and after 21 days of treatment (V2): Reflex volume served as primary, leak point volume and maximum detrusor pressure as secondary efficacy outcomes, treatment-related adverse events as tolerability outcomes.

Results: Sixty-six patients with proven NDO were enrolled. Reflex volume (ml) increased significantly in the IR (V1: 100.9, V2: 202.9) and in the ER (V1: 89.8, V2: 180.3) group, no significant intergroup difference. Leak point volume increased, and maximum detrusor pressure decreased significantly in both groups, no significant intergroup differences. The percentage of patients presenting with incontinence was reduced by $14 \%$ in the IR and by 39\% in the ER group, the difference is significant. Treatment-related adverse events manifested in 42 and $36 \%$ following propiverine IR and ER, respectively.

Conclusion: The urodynamic efficacy outcomes demonstrated both galenic formulations to be equieffective. However, following propiverine ER $45 \mathrm{mg}$ s.i.d. higher continence rates compared with propiverine IR $15 \mathrm{mg}$ t.i.d. were achieved, possibly indicative of more balanced plasma-levels. A slight tendency for superior tolerability outcomes of propiverine ER compared with IR was demonstrated.

Sponsorship: The study was sponsored by an unrestricted educational grant of APOGEPHA Arzneimittel GmbH, Dresden, Germany. Spinal Cord (2013) 51, 419-423; doi:10.1038/sc.2012.174; published online 22 January 2013

Keywords: spinal cord injury; neurogenic detrusor overactivity; antimuscarinics; propiverine; adults

\section{INTRODUCTION}

Antimuscarinics are widely used in the treatment of overactive bladder (OAB)/ idiopathic detrusor overactivity (IDO), and neurogenic detrusor overactivity (NDO). However, only immediate-release (IR) formulations of oxybutynin, ${ }^{1-3}$ propiverine, ${ }^{3,4}$ and trospium chloride ${ }^{2,5}$ were approved in NDO so far. ${ }^{6}$ Extended-release (ER) formulations of antimuscarinics were developed assuming that more stable plasma concentrations will introduce less variation in efficacy over time and enhance treatment compliance. The relatively stable serum concentration is expected to improve the tolerability by avoiding the high peaks, which can be associated with an increased incidence of (generally minor) adverse events, as shown in pharmacokinetic studies of oxybuytnin ${ }^{7}$ and tolterodine. ${ }^{8}$

By introducing ER formulations for treatment in OAB/ IDO, for example, oxybutynin ER, ${ }^{9}$ propiverine ER $30 \mathrm{mg},{ }^{10}$ tolterodine ER $4 \mathrm{mg},{ }^{11}$ and trospium chloride ER $60 \mathrm{mg},{ }^{12}$ improved outcomes were reported. These studies reported comparable efficacy combined with an improved tolerability profile for ER formulations in OAB/ IDO. However, clinical results with respect to ER formulations of antimuscarinics in adults suffering from NDO have not been published so far. For the ER formulation of propiverine hydrochloride (in the following abbreviated as propiverine), additional rationales have to be taken into account: not only a slower drug release, but also a reduced formation of active metabolites in the colon. Moreover, CYP3A4 and ABCC2, the major variables in pharmacokinetics of propiverine, are less expressed in the colon, the predominant absorption site for propiverine ER, whereas propiverine IR is mainly absorbed in the proximal small intestine. ${ }^{13}$ The galenic properties of the ER formulation of propiverine have been published recently. ${ }^{14}$

Our aim in this study was to evaluate the potential benefits of propiverine ER $45 \mathrm{mg}$ s.i.d. compared with propiverine IR $15 \mathrm{mg}$ t.i.d. with respect to efficacy, tolerability and patient convenience in patients suffering from NDO.

\footnotetext{
${ }^{1}$ Department of Urology and Paediatric Urology, Universität Essen, Essen, Germany; ${ }^{2}$ APOGEPHA Arzneimittel GmbH, Dresden, Germany; ${ }^{3}$ Bennebroek, Holland; ${ }^{4}$ Urological Practice, Hagenow, Germany; ${ }^{5}$ Department of Urology, Medical University Graz, Austria; ${ }^{6}$ Prof. Dr. Th. Burghele Clinical Hospital, Bucharest, Romania; ${ }^{7}$ Department of Urology, Geroia Hospital, Bucharest, Romania and ${ }^{8}$ Uro-Andro-Med, Bucharest, Romania

Correspondence: Professor M Stöhrer, Department of Urology and Paediatric Urology, Universität Essen, Hufelandstr. 55, Essen 45122, Germany.

E-mail: manfred@stoehrer.com
}

Received 7 June 2012; revised 19 November 2012; accepted 10 December 2012; published online 22 January 2013 


\section{MATERIALS AND METHODS}

\section{Study design}

The phase III study was conducted in a double-blind, double-dummy, randomized, parallel group design at six European study centers, all specialised in neurourology. Study conduct was in accordance to the declaration of Helsinki and Good Clinical Practice Guidelines, patients gave written informed consent. The study is registered in clinical trial registers (EudraCT 2004001275-19; ClinicalTrials.gov NCT01530620). Institutional and governmental regulations concerning the ethical use of patients were followed.

Propiverine was administered either in capsules (ER $45 \mathrm{mg}$ s.i.d.) or in coated tablets (IR $15 \mathrm{mg}$ t.i.d.), using the double-dummy technique. Concomitant medication interfering with the trial medication was not allowed and had to be terminated at least 7 days before study start. The key inclusion criteria were: (i) female or male Caucasian patients $\geqslant 18$ and $\leqslant 70$ years of age; (ii) NDO proven as occurrence of reflex detrusor contractions; (iii) reflex volume $^{15}$ of $\leqslant 250 \mathrm{ml}$. The key exclusion criteria comprised: (i) unstable multiple sclerosis within the last 3 months; (ii) increased post void residual $(\geqslant 20 \%$ of the maximum bladder capacity and self-catheterization not possible); (iii) acute urinary tract infection; (iv) anomalies, radiation or surgery of the lower urinary tract; (v) contraindications for antimuscarinics; (vi) cardiac insufficiency; (vii) botulinum toxin treatment within the last 12 months.

\section{Outcomes}

Urodynamic and clinical outcome parameters were assessed at baseline (V1) and after 21 days of treatment (V2). The urodynamic parameters were assessed following the definitions of the International Continence Society. ${ }^{15,16}$

The change from V1 to V2 in reflex volume served as primary efficacy outcome. In case of non-occurrence of uninhibited detrusor contractions during filling cystometry, reflex volume was imputed by maximum cystometric capacity.

Secondary efficacy outcomes were the maximum detrusor pressure and the leak point volume, the latter defined as infused volume at first leakage. In case of non-occurrence of leakage during filling cystometry, leak point volume was imputed by maximum cystometric capacity as a conservative measurement.

All urodynamic parameters were assessed by the investigators of the study centers, and by an independent reviewer, highly experienced in evaluating urodynamic results. The data differed not relevantly, irrespective of the investigated parameter. The reviewer's values were taken as results to guarantee a standardized analysis across all study centers.

Incontinence was assessed by asking the patients about the number of episodes per $24 \mathrm{~h}$.

Adverse events, their intensity ('mild', 'moderate', 'severe'), and their relationship to the trial medication ('certain', 'probable', 'possible', 'unlikely', 'conditional', 'not assessable') were assessed as tolerability outcomes. A final evaluation of the tolerability of the medication by the investigators and the patients was conducted at the end of the study period according to the four categories 'very good', 'good', 'moderate' and 'poor'.

Post void residual urine was determined in patients with spontaneous voiding.

\section{Statistical analysis}

The primary efficacy outcome was defined as change in reflex volume from V1 to V2 in the per-protocol-population. An analysis of covariance model with treatment groups and baseline values as explanatory variables was performed. Non-inferiority of propiverine ER compared with propiverine IR was tested with a non-inferiority margin of $\leqslant 25 \mathrm{ml}$. A two-stage adaptive test design with interim analysis after enrollment of 60 patients was planned with an overall one-sided significance level of $\alpha=0.025$. If necessary, this approach allows for sample size adjustments during the second recruitment period or for premature discontinuation of the study (confidence interval 95\%). The secondary urodynamic outcome parameters in the intention-to-treat- and in the per-protocol-population were also analyzed by applying the analysis of covariance model. Furthermore, all efficacy parameters were analyzed by using descriptive statistics.

\section{RESULTS}

\section{Patient population characteristics}

The allocation of the 66 (33 in each arm) enrolled patients with respect to the safety-, intention-to-treat-, and per-protocol-population is given in Table 1, demographic and baseline characteristics in Tables 2 and 3. All patients, except one in each group, were treatmentnaïve with treatments longer than two years ago. Before study initiation in the IR and the ER group, respectively, patients initiated voiding spontaneously (22 vs 22 ), by abdominal straining/ triggering ( 5 vs 8 ), by indwelling or intermittent catheterization ( 5 vs 2 ) or by other methods (0 vs 2). During study conduct all patients were trained in intermittent self catheterization.

\section{Primary efficacy outcome parameter}

Reflex volume improved significantly by $102.0 \pm 85.2 \mathrm{ml}$ in the propiverine IR and by $90.5 \pm 92.1 \mathrm{ml}$ in the ER group, intergroup differences were non-significant (Table 4). The mean treatment group difference was $-12.4 \mathrm{ml}$ with a $95 \%$ confidence interval ranging from -58.9 to $34.0 \mathrm{ml}$. The one-sided $P$-value for the test against the hypothesis 'ER-IR $\leqslant-25 \mathrm{ml}$ ' was 0.2952 . This $P$-value was in the

\section{Table 1 Allocation of patient populations (\%)}

\begin{tabular}{lccc}
\hline & $\begin{array}{c}\text { Immediate-release } \\
(\mathrm{N}=33)\end{array}$ & $\begin{array}{c}\text { Extended-release } \\
(\mathrm{N}=33)\end{array}$ & $\begin{array}{c}\text { Total } \\
(\mathrm{N}=66)\end{array}$ \\
\hline $\begin{array}{l}\text { Randomised } \\
\begin{array}{l}\text { Included in safety } \\
\text { population } \\
\quad \text { Excluded from ITT } \\
\text { population } \\
\text { Lost to follow-up after } \\
\text { visit 1 }\end{array}\end{array}$ & $13(100)$ & $33(100)$ & $66(100)$ \\
$\begin{array}{l}\text { Included in ITT } \\
\text { population } \\
\text { Excluded from PP } \\
\text { population }\end{array}$ & $1(3)$ & $33(100)$ & $66(100)$ \\
$\begin{array}{l}\text { Included in PP } \\
\text { population }\end{array}$ & $32(97)$ & $0(0)$ & $1(2)$ \\
\hline
\end{tabular}

Abbreviations: PP, per protocol; ITT, intention to treat.

Table 2 Demographic characteristics of the patient population

\begin{tabular}{llccc}
\hline Variable & $\begin{array}{c}\text { Category/ } \\
\text { statistic }\end{array}$ & $\begin{array}{c}\text { Immediate- } \\
\text { release }(\mathrm{N}=33)\end{array}$ & $\begin{array}{c}\text { Extended-release } \\
(\mathrm{N}=33)\end{array}$ & $\begin{array}{c}\text { Total } \\
(\mathrm{N}=66)\end{array}$ \\
\hline Sex, $n(\%)$ & Male & $22(67)$ & $19(58)$ & $41(62)$ \\
& Female & $11(33)$ & $14(42)$ & $25(38)$ \\
Age, years & $N$ & 33 & 33 & 66 \\
& Mean (s.d.) & $41.4(16.7)$ & $40.9(16.9)$ & $41.2(16.7)$ \\
& Median & 36.0 & 35.0 & 35.5 \\
Age group, $n$ (\%) & $18-39$ yrs & $19(58)$ & $17(52)$ & $36(55)$ \\
& $40-64$ yrs & $10(30)$ & $12(36)$ & $22(33)$ \\
& $65-75$ yrs & $4(12)$ & $4(12)$ & $8(12)$ \\
Body-mass & $N$ & 33 & 33 & 66 \\
index, kg m ${ }^{-2}$ & Mean (s.d.) & $23.4(3.6)$ & $23.5(4.3)$ & $23.4(3.9)$ \\
& Median & 22.5 & 23.3 & 22.8
\end{tabular}

SD standard deviation

Abbreviation: Yrs, years. 
a priori defined range $(0.0102<P$-value $<0.5)$, for which the interim analysis neither showed non-inferiority nor futility to demonstrate non-inferiority.

Assuming a significance level of $\alpha_{2}=0.013$ for the second period of patient enrollment according to the applied algorithm and $\beta=0.2$ as

Table 3 Baseline characteristics of the intention to treat population

\begin{tabular}{|c|c|c|c|c|}
\hline Variable & $\begin{array}{l}\text { Category/ } \\
\text { statistic }\end{array}$ & $\begin{array}{l}\text { Immediate- } \\
\text { release } \\
(\mathrm{N}=32)\end{array}$ & $\begin{array}{c}\text { Extended- } \\
\text { release } \\
(\mathrm{N}=33)\end{array}$ & $\begin{array}{l}\text { Total } \\
(\mathrm{N}=65)\end{array}$ \\
\hline $\begin{array}{l}\text { Time since manifestation of } \\
\text { neurogenic impact, years }\end{array}$ & $\begin{array}{l}N \\
\text { Mean (s.d.) } \\
\text { Median }\end{array}$ & $\begin{array}{l}32 \\
7.5(7.9) \\
5.3\end{array}$ & $\begin{array}{l}33 \\
5.4(4.2) \\
4.2\end{array}$ & $\begin{array}{l}65 \\
6.5(6.4) \\
5.1\end{array}$ \\
\hline $\begin{array}{l}\text { Type of neurological } \\
\text { impact, } n(\%)\end{array}$ & $\begin{array}{l}\text { Traumatic } \\
\text { Stroke } \\
\text { Inflammable } \\
\text { Degenerative }\end{array}$ & $\begin{aligned} & 16(50) \\
& 8(25) \\
& 7(22) \\
& 1(3)\end{aligned}$ & $\begin{aligned} 21 & (64) \\
7 & (21) \\
5 & (15) \\
0 & (0)\end{aligned}$ & $\begin{array}{c}37(57) \\
15(23) \\
12(18) \\
1(2)\end{array}$ \\
\hline $\begin{array}{l}\text { Level of neurological } \\
\text { impact, } n(\%)\end{array}$ & $\begin{array}{l}\text { Suprasacral } \\
\text { Suprapontine } \\
\text { Suprapontine } \\
\text { and Suprasacral }\end{array}$ & $\begin{array}{c}23(72) \\
9(28) \\
0(0)\end{array}$ & $\begin{array}{c}24(73) \\
8(24) \\
1(3)\end{array}$ & $\begin{array}{c}47(72) \\
17(26) \\
1(2)\end{array}$ \\
\hline $\begin{array}{l}\text { Frequency of micturition/ } \\
\text { catheterisation (per } 24 \mathrm{~h} \text { ) }\end{array}$ & $\begin{array}{l}N \\
\text { Mean (s.d.) } \\
\text { Median }\end{array}$ & $\begin{array}{c}32 \\
13.8(7.7) \\
12.5\end{array}$ & $\begin{array}{c}33 \\
12.2(5.7) \\
12.0\end{array}$ & $\begin{array}{c}65 \\
13.0(6.8) \\
12.0\end{array}$ \\
\hline
\end{tabular}

type II error rate, altogether 938 evaluable patients per group (that is, per-protocol) would have to be included after interim analysis. This estimated number of almost 2000 patients and the low incidence rate of NDO in the general population ${ }^{6}$ impeded study continuation in a realistic time and budget frame, resulting in premature termination of the study.

\section{Secondary efficacy outcome parameters}

Leak point volume increased and maximum detrusor pressure decreased significantly in both treatment groups without significant intergroup differences (Table 4).

Most interestingly, the improvements of all key urodynamic parameters, calculated additionally as change in percentage for preand post-treatment values, are almost identical across both treatment groups (Table 4).

\section{Continence}

The number of patients presenting with incontinence was reduced in the propiverine IR group from 23 to 19 patients, and in the ER group from 25 to 13 patients (Table 4). These results are indicative of statistical significance in the propiverine ER group only and, moreover, of a statistical significant intergroup difference.

\section{Tolerability}

Sixteen patients in the propiverine IR group and 12 patients in the ER group experienced at least one adverse event (Table 5). Treatment-

Table 4 Urodynamic and clinical parameters of the per protocol population

\begin{tabular}{|c|c|c|c|c|}
\hline Endpoint & Category & Statistic & Immediate-release $(\mathrm{N}=29)$ & Extended-release $(\mathrm{N}=31)$ \\
\hline \multirow[t]{9}{*}{ Reflex volume (ml) } & \multirow[t]{2}{*}{ Baseline (V1) } & Mean (s.d.) & $100.9(74.4)$ & $89.8(61.4)$ \\
\hline & & Median & 89.0 & 88.0 \\
\hline & \multirow[t]{2}{*}{ Follow-up (V2) } & Mean (s.d.) & $202.9(112.1)$ & $180.3(104.7)$ \\
\hline & & Median & 213.0 & 161.0 \\
\hline & \multirow[t]{2}{*}{$\mathrm{V} 2-\mathrm{V} 1$} & Mean (s.d.) & $102.0(85.2)$ & $90.5(92.1)$ \\
\hline & & Median & 103.0 & 78.0 \\
\hline & \multicolumn{2}{|l|}{ Student's $t$-test $P$-value } & $<0.0001$ & $<0.0001$ \\
\hline & \multicolumn{2}{|l|}{ ANCOVA $P$-value } & 0.59 & \\
\hline & \multicolumn{2}{|l|}{ V2 -V1 (\%) } & $+101 \%$ & $+101 \%$ \\
\hline \multirow[t]{9}{*}{ Leak point volume (ml) } & \multirow[t]{2}{*}{ Baseline (V1) } & Mean (s.d.) & $124.1(75.3)$ & $106.7(54.6)$ \\
\hline & & Median & 120.0 & 95.0 \\
\hline & \multirow[t]{2}{*}{ Follow-up (V2) } & Mean (s.d.) & $228.6(111.3)$ & $204.3(101.7)$ \\
\hline & & Median & 250.0 & 173.0 \\
\hline & \multirow[t]{2}{*}{$\mathrm{V} 2-\mathrm{V} 1$} & Mean (s.d.) & $104.4(74.8)$ & $97.5(87.7)$ \\
\hline & & Median & 105.0 & 86.0 \\
\hline & \multicolumn{2}{|l|}{ Student's $t$-test $P$-value } & $<0.0001$ & $<0.0001$ \\
\hline & \multicolumn{2}{|l|}{ ANCOVA $P$-value } & 0.78 & \\
\hline & \multicolumn{2}{|l|}{ V2 -V1 (\%) } & $+84.2 \%$ & $+91.5 \%$ \\
\hline \multirow{9}{*}{$\begin{array}{l}\text { Maximum detrusor pressure } \\
\left(\mathrm{cmH}_{2} \mathrm{O}\right)\end{array}$} & \multirow[t]{2}{*}{ Baseline (V1) } & Mean (s.d.) & $66.1(42.3)$ & $67.0(31.1)$ \\
\hline & & Median & 59.0 & 63.0 \\
\hline & \multirow[t]{2}{*}{ Follow-up (V2) } & Mean (s.d.) & $42.4(25.4)$ & $43.7(24.5)$ \\
\hline & & Median & 42.0 & 42.0 \\
\hline & \multirow[t]{2}{*}{$\mathrm{V} 2-\mathrm{V} 1$} & Mean (s.d.) & $-23.8(41.2)$ & $-23.3(27.2)$ \\
\hline & & Median & -20.0 & -20.0 \\
\hline & \multicolumn{2}{|l|}{ Student's $t$-test $P$-value } & 0.0043 & $<0.0001$ \\
\hline & \multicolumn{2}{|l|}{ ANCOVA $P$-value } & 0.86 & \\
\hline & \multicolumn{2}{|l|}{ V2 -V1 (\%) } & $-35.9 \%$ & $-34.8 \%$ \\
\hline \multirow[t]{5}{*}{ Incontinence $(N ; \%)$} & \multicolumn{2}{|l|}{ Baseline (V1) } & $23(79)$ & $25(81)$ \\
\hline & \multicolumn{2}{|l|}{ Follow-up (V2) } & $19(66)$ & $13(42)$ \\
\hline & \multicolumn{2}{|l|}{ Student's $t$-test $P$-value } & 0.125 & 0.0005 \\
\hline & \multicolumn{2}{|l|}{ ANCOVA $P$-value } & 0.041 & \\
\hline & \multicolumn{2}{|l|}{ V2-V1 (\%) } & -14 & -39 \\
\hline
\end{tabular}

Abbreviation: ANCOVA, analysis of covariance. 
Table 5 Number of patients with adverse events by MedDRA system organ class and preferred term (safety population) ${ }^{\mathrm{a}}$

\begin{tabular}{|c|c|c|c|c|}
\hline \multirow{3}{*}{$\begin{array}{l}\text { MedDRA system organ class preferred } \\
\text { term, } \mathrm{n}(\%) \\
\text { All patients }\end{array}$} & \multicolumn{2}{|c|}{ Immediate-release } & \multicolumn{2}{|c|}{ Extended-release } \\
\hline & Overall & Related $^{\mathrm{b}}$ & Overall & Related $^{\mathrm{b}}$ \\
\hline & \multicolumn{2}{|c|}{$N=33$} & \multicolumn{2}{|c|}{$N=33$} \\
\hline Any adverse event & $16(48)$ & $14(42)$ & $12(36)$ & $12(36)$ \\
\hline Gastrointestinal disorders & $11(33)$ & $11(33)$ & $10(30)$ & $10(30)$ \\
\hline Dry mouth & $8(24)$ & $8(24)$ & $9(27)$ & $9(27)$ \\
\hline Gastrointestinal motility disorder & $3(9)$ & $3(9)$ & $1(3)$ & $1(3)$ \\
\hline Abdominal pain & $0(0)$ & $0(0)$ & $1(3)$ & $0(0)$ \\
\hline Nausea & $0(0)$ & $0(0)$ & $1(3)$ & $0(0)$ \\
\hline Nervous system disorders & $2(6)$ & $0(0)$ & $3(9)$ & $3(9)$ \\
\hline Dizziness & $0(0)$ & $0(0)$ & $3(9)$ & $3(9)$ \\
\hline Headache & $2(6)$ & $0(0)$ & $0(0)$ & $0(0)$ \\
\hline Eye disorders & $2(6)$ & $2(6)$ & $0(0)$ & $0(0)$ \\
\hline Accommodation disorder & $1(3)$ & $1(3)$ & $0(0)$ & $0(0)$ \\
\hline Vision blurred & $1(3)$ & $1(3)$ & $0(0)$ & $0(0)$ \\
\hline $\begin{array}{l}\text { General disorders and administration } \\
\text { site conditions }\end{array}$ & $1(3)$ & $0(0)$ & $1(3)$ & $0(0)$ \\
\hline Pyrexia & $1(3)$ & $0(0)$ & $1(3)$ & $0(0)$ \\
\hline Renal and urinary disorders & $1(3)$ & $1(3)$ & $0(0)$ & $0(0)$ \\
\hline Dysuria & $1(3)$ & $1(3)$ & $0(0)$ & $0(0)$ \\
\hline
\end{tabular}

${ }^{a}$ Counts are for patients, not for events

${ }^{b}$ Adverse events with certain, probable, possible, conditional or unassessable relationship to study medication

related adverse events manifested in 14 patients in the IR and in 12 patients in the ER group. With respect to severity, four patients in the IR group and two patients in the ER group experienced a severe adverse event, out of which two in the IR group and one in the ER group were treatment-related. Serious adverse events or adverse events necessitating dose reductions or premature withdrawal of medication did not manifest in either treatment group.

Overall tolerability was assessed both by investigators and patients. In general, the patients rated the tolerability worse in comparison to the investigators. In the propiverine IR and ER group, respectively, $23(72 \%)$ vs $26(79 \%)$ of the patients rated the tolerability as 'very good' or 'good', $9(28 \%)$ vs $7(21 \%)$ of the patients as 'moderate' or 'poor'.

Post void residual urine increased, clinically not relevant by only $17.6 \pm 34.2 \mathrm{ml}$ (V1: $9.7 \pm 10.9, \mathrm{~V} 2: 27.3 \pm 37.5)$ in the propiverine IR group and by $17.0 \pm 31.3 \mathrm{ml}(\mathrm{V} 1: 12.0 \pm 29.5$, V2: $28.9 \pm 36.6)$ in the propiverine ER group.

\section{DISCUSSION}

The primary objective, demonstrating non-inferiority of propiverine ER compared with propiverine IR, was not achieved in this study. This is mainly due to the limited number of patients enrolled, reflecting that NDO is a rare disease. Consecutively, the recruitment of almost 2000 patients, as requested according to the interim analysis, was not feasible. Nevertheless, the primary efficacy parameter, reflex volume and the secondary efficacy parameters, leak point volume and maximum detrusor pressure, demonstrated statistically significant and clinically relevant improvements for both galenic formulations. Interestingly, almost identical pressure reductions by $\sim 23 \mathrm{~cm} \mathrm{H} H_{2} \mathrm{O}$ were achieved in both treatment groups for this key urodynamic parameter, used in most placebo- and active-controlled studies as key efficacy outcome..$^{2-5}$ This reduction in maximum detrusor pressure is comparable with reductions by $\sim 19$ and $27 \mathrm{~cm}$ $\mathrm{H}_{2} \mathrm{O}$ administering propiverine $15 \mathrm{mg}$ t.i.d. ${ }^{3,4}$ We consider these outcomes as real treatment results as in all placebo-groups of controlled studies in NDO no placebo effects manifested at all. ${ }^{4,5}$ The fact that no placebo effect is found in patients with NDO, contrary to patients with $\mathrm{OAB} / \mathrm{IDO}$, has probably two causes: (1). the stability of the underlying disease during the limited treatment periods and (2). the obviously missing, most probably psychologically mediated, placebo improvements. Therefore, from a clinical point of view it can be concluded that the key objective in our patient population, protecting the upper urinary tract by the 'conversion of an overactive, high-pressure bladder into a lowpressure reservoir' ${ }^{6}$ will be achieved under clinical conditions by both galenic formulations.

The urodynamically assessed bladder capacity in terms of reflex volume and leak point volume are interdependent. An increase by 90-100 ml, both for reflex volume and leak point volume, following either propiverine IR or ER, is in accordance with an increase by $\sim 104^{4}$ and $110 \mathrm{ml}^{3}$ for cystometric bladder capacity following propiverine $15 \mathrm{mg}$ t.i.d. Moreover, the improvements in these key parameters, paralleled by a substantial decrease in maximum detrusor pressure, confirm previous results for propiverine, oxybutynin and trospium chloride, the antimuscarinics most intensively investigated in patients with NDO. ${ }^{1-5}$

One of the most striking findings of this study is the significantly higher percentage of patients achieving continence in the propiverine ER compared with the IR group. We hypothesize that the clinical parameter incontinence, contrary to urodynamic parameters, might reflect to a greater extent the clinical response to more balanced plasma concentrations of propiverine. Both the efficacy outcomes, documented in improved continence rates, and a slight tendency of a superior tolerability profile of propiverine ER compared with IR, give hints for this assumption. However, the interrelationship between pharmacokinetics and pharmacodynamics was not elucidated in this study: determination of the plasma-levels of the active ingredient propiverine and its metabolites would have made this study more invasive than was feasible.

Taking into account the pharmacological profile of propiverine it can be assumed that metabolites, possessing strong antimuscarinic properties, have contributed to the efficacy of propiverine: antimuscarinics are especially effective in $\mathrm{NDO}$, as the contribution of cholinergic transmission to detrusor contraction is considerably increased, whereas the purinergic component is decreased in comparison to $\mathrm{OAB} / \mathrm{IDO} .{ }^{17}$ Additionally, serum concentrations of the main metabolite M-5 are significantly reduced by about $10 \%$ during the treatment with propiverine ER..$^{13}$ Due to the lower intrinsic activity of M-5 in comparison with propiverine, and their different modes of action ${ }^{18}$ this may result in improvements of the safety and efficacy, as was speculated earlier. ${ }^{13}$ Therefore, smoothening of serum concentrations by using ER dosage forms is a suitable concept to further improve the tolerability of propiverine, as shown for oxybutynin ${ }^{7}$ and tolterodine. ${ }^{8}$

In conclusion, the expectation of further optimizing treatment outcomes by introducing a $45 \mathrm{mg}$ ER formulation of propiverine was fulfilled: Clinically comparable efficacy of both formulations associated with a slight tendency of improved tolerability of the ER compared with the IR formulation is documented in the overall rate of adverse events, the rate of treatment-related adverse events, and in the tolerability assessments (Table 5). Both in patients with NDO and in those patients with $\mathrm{OAB} / \mathrm{IDO}$, in which higher antimuscarinic 
doses are necessitated, propiverine ER $45 \mathrm{mg}$ will enlarge the therapeutic armamentarium. In consistency with the presented study results a more favorable tolerability profile of the $30 \mathrm{mg}$ ER compared with the $15 \mathrm{mg}$ b.i.d. IR formulation of propiverine was already demonstrated in patients suffering from OAB. ${ }^{10}$

Future research should address, whether ER compared with IR formulations of propiverine are superior with respect to the following issues: (1) the superior continence rates following propiverine ER compared with IR formulations need to be further elucidated. (2) Unfortunately, pharmacoeconomic studies of antimuscarinics focussing on NDO have not yet been published. However, in patients with $\mathrm{OAB}$ pharmacoeconomic studies have shown more favorable patient compliance $(74.3$ vs $60.9 \%)$ and adherence rates (115 vs 60 days) following ER compared with IR formulations of antimuscarinics. ${ }^{19}$ Other studies confirmed pharmacoeconomic advantages of ER compared with IR formulations in $\mathrm{OAB}$ patients for treatment persistence over 1 year ( 15.3 vs $6.5 \%$ ), switch rates of 16.5 and $19.4 \%$, and adherence rates of 36.1 and $14.8 \%$ to antimuscarinics, respectively. ${ }^{20}$ (3) The merits of a propiverine ER formulation applied once-daily and its beneficial impact on improved patient convenience and quality of life still need to be proven. These issues are of paramount importance, especially in patients suffering from NDO requiring life-long medication in order to avoid lethal sequelae.

\section{DATA ARCHIVING}

There were no data to deposit.

\section{CONFLICT OF INTEREST}

The study was sponsored by an unrestricted educational grant of APOGEPHA Arzneimittel GmbH, Dresden, Germany. G. Mürtz is a medical consultant, GS is an employee of APOGEPHA.

\section{ACKNOWLEDGEMENTS}

We thank Karin Billy for providing some of the references; and Barbara Tegelkamp for technical assistance in supporting the manuscript. We also thank Frieder Schnabel, who was in lead for the statistical study planning, and analysis. Unfortunately, he died much too early at an age of 61 years. We commemorate that Frieder Schnabel has accomplished his outstanding mission by giving statistical guidance to a study highly dependent on this expertise. We also acknowledge valuable support given by Florian Schirm, DATAMAP, Freiburg, Germany, with respect to planning, conduct, and finalization of this study.
1 Gajewski JB, Awad SA. Oxybutynin versus propantheline in patients with multiple sclerosis and detrusor hyperreflexia. J Urol 1986; 135: 966-968.

2 Madersbacher $\mathrm{H}$, Stöhrer M, Richter R, Burgdörfer H, Hachen HJ, Mürtz G. Trospium chloride versus oxybutynin: a randomized, double-blind, multicentre trial in the treatment of detrusor hyperreflexia. Br J Urol 1995; 75: 452-456.

3 Stöhrer M, Mürtz G, Kramer G, Schnabel F, Arnold EP, Wyndaele JJ et al. Propiverine compared to oxybutynin in neurogenic detrusor overactivity - results of a randomized double-blind, multicenter study. Eur Urol 2007; 52: 235-242.

4 Stöhrer M, Madersbacher H, Richter R, Wehnert J, Dreikorn K. Efficacy and safety of propiverine in $\mathrm{SCl}$-patients suffering from detrusor hyperreflexia - a double-blind, placebo-controlled clinical trial. Spinal Cord 1999; 37: 196-200.

5 Stöhrer M, Bauer P, Giannetti BM, Richter R, Burgdörfer H, Mürtz G. Effect of trospium chloride on urodynamic parameters in patients with detrusor hyperreflexia due to spinal cord injuries. A multicentre placebo-controlled double-blind trial. Urol Int 1991; 47: 138-143.

6 Pannek J, Stöhrer M, Blok B, Castro-Diaz D, Del Popolo G, Kramer G et al. Guidelines on neurogenic lower urinary tract dysfunction. European Association of Urology 2011, pp 1-64.

7 Siddiqui MA, Perry CM, Scott LJ. Oxybutynin extended release: a review of its use in the management of overactive bladder. Drugs 2004; 64: 885-912.

8 Olsson B, Szamosi J. Multiple dose pharmacokinetics of a new once daily extended release tolterodine formulation versus immediate release tolterodine. Clin Pharmacokinet 2001; 40: 227-235.

9 Anderson RU, Mobley D, Blank B, Saltzstein D, Susset J, Brown JS. Once daily controlled versus immediate release oxybutynin chloride for urge urinary incontinence. J Urol 1999; 161: 1809-1812.

10 Jünemann KP, Hessdörfer E, Unamba-Oparah I, Berse M, Brünjes R, Madersbacher H et al. Propiverine hydrochloride immediate and extended release: comparison of efficacy and tolerability in patients with overactive bladder. Urol Int 2006; 77: 334-339.

11 Van Kerrebroeck P, Kreder K, Jonas U, Zinner N, Wein A et al. Tolterodine once-daily: superior efficacy and tolerability in the treatment of overactive bladder. Urology 2001; 57: 414-421.

12 Dmochowski R, Sand P, Zinner N, Staskin D. Trospium chloride once daily (QD) for overactive bladder syndrome: results from a placebo-controlled interventional study. Urology 2008; 71: 449-454.

13 May K, Westphal K, Giessmann T, Wegner D, Adam U, Lerch MM et al. Disposition and antimuscarinic effects of the urinary bladder spasmolytics propiverine: influence of dosage forms and circadian-time rhythms. J Clin Pharm 2008; 48: 570-579.

14 Ploen J, Andersch J, Heschel M, Leopold S. Citric acid as pH-modifying additive in an extended releasepellet formulation containing a weakly basic drug. Drug Dev Ind Pharm 2009; 35: 1210-1218.

15 Stöhrer M, Goepel M, Kondo A, Kramer G, Madersbacher H, Millard R et al. The standardization of terminology in neurogenic lower urinary tract dysfunction: with suggestions for diagnostic procedures. International Continence Society Standardization Committee. Neurourol Urodyn 1999; 18: 139-158.

16 Abrams P, Cardozo L, Fall M, Griffiths D, Rosier P, Ulmsten U et al. The standardisation of terminology of lower urinary tract function: report from the standardisation sub-committee of the International Continence Society. Neurourol Urodyn 2002; 21: 167-178.

17 Yokota T, Yamaguchi $\mathrm{O}$. Changes in cholinergic and purinergic neurotransmission in pathologic bladder of chronic spinal rabbit. J Urol 1996; 156: 1862-1866.

18 Wuest M, Hecht J, Christ T, Braeter M, Schoeberl C, Hakenberg OW et al. Pharmacodynamics of propiverine and three of its main metabolites on detrusor contraction. Br J Pharmacol 2005; 145: 608-619.

19 Varadharajan S, Jumadilova Z, Girase P, Ollendorf D. Economic impact of extendedrelease tolterodine versus immediate- and extended-release oxybutynin among commercially insured persons with overactive bladder. Am J Managed Care 2005; 11: S140-S149.

20 D'Souza A, Smith M, Miller L-A, Doyle J, Ariely R. Persistence, adherence, and switch rates among extended-release and immediate-release overactive bladder medications in a regional managed care plan. J Managed Care Pharm 2008; 14: 291-301. 